\title{
Dyspnea, massive effusion and lytic rib lesion as initial presentation of multiple myeloma in a young man
}

\author{
Mohammed H AlShati MD FRCPC ${ }^{1}$, Ramesh Kumar MD PhD ${ }^{2}$, Shreeram Kannan MD FRCR(UK) ${ }^{3}$
}

\begin{abstract}
MH AlShati, R Kumar, S Kannan. Dyspnea, massive effusion and lytic rib lesion as initial presentation of multiple myeloma in a young man. Can Respir J 2013;20(4):253-255.

Multiple myeloma, a disorder commonly encountered in elderly patients, represents a malignant proliferation of plasma cells that primarily affects bone marrow. Pleural effusion as the presenting manifestation of the disease is uncommon. The authors report a case of multiple myeloma with unusual features presenting at a relatively young age with massive spontaneous hemothorax and multiple thoracic masses completely obscuring rib shadow on plain chest imaging. The patient demonstrated a good response to melphalan chemotherapy without recurrence of effusion or the need for additional chemical or surgical pleural interventions.
\end{abstract}

Key Words: Absent rib shadow; Multiple myeloma; Spontaneous hemothorax
Learning objectives:

-To recognize the different etiologies of pleural effusion in multiple myeloma (MM).

- To understand that pleural and/or chest wall abnormalities can occasionally be the presenting manifestation of hematological malignancy.

\section{CanMEDS competency: Medical Expert}

\section{Pretest:}

1. What are the causes of pleural effusion in MM?

2. Which malignant conditions commonly affect the ribs?

MM, a disorder commonly encountered in elderly patients, represents a malignant proliferation of plasma cells that chiefly affects bone marrow. The most frequent thoracic involvement with $\mathrm{MM}$ is apparent in the form of diffuse bony lesions or pulmonary infiltrates secondary to an infectious process (1). Presentation with massive unilateral spontaneous hemothorax and absent rib shadow on plain chest imaging, especially at a young age, is unique.

\section{CASE PRESENTATION}

A 32-year-old nonsmoking Asian man presented to the emergency department with subacute left-sided chest pain followed by progressive shortness of breath. The pain was mostly posterior, did not radiate and lacked pleuritic characteristics. There was no history of chest trauma, cough, fever, weight loss, recent travel, substance abuse or HIV risk factors. Family and environmental history were unremarkable. On physical examination, he was afebrile, exhibited mild tachypnea and tachycardia, appeared pale and showed no clubbing. His blood pressure was normal. A chest examination revealed complete dullness over the left hemithorax with absent breath sounds; oxygen saturation on room air, however, was $94 \%$. The remainder of his examination was unremarkable.

Except for mild anemia (hemoglobin level $90 \mathrm{~g} / \mathrm{L}$ ), a complete blood count was unremarkable. A biochemical profile showed normal serum calcium and electrolyte levels, normal hepatic and renal status,

\section{Un mélyome multiple à la présentation initiale de dyspnée, d'effusion massive et de lésion costale lytique chez un jeune homme}

Le myélome multiple, un trouble fréquent chez les patients âgés, est une prolifération maligne de plasmocytes qui touche principalement la moelle osseuse. Il se manifeste souvent sous forme d'effusion pleurale. Les auteurs signalent le cas d'un myélome multiple aux caractéristiques inhabituelles, se manifestant chez une personne relativement jeune sous forme d'hémothorax spontané massif et de multiples masses thoraciques obscurcissant totalement l'ombre costale à l'imagerie simple du thorax. Le patient a bien répondu à une chimiothérapie au melphalane, sans récurrence de l'effusion ou autres interventions pleurales de nature chimique ou chirurgicale. and normal total protein, albumin, uric acid and lactate dehydrogenase levels. Urine testing revealed no abnormality. Modest elevation of erythrocyte sedimentation rate $(69 \mathrm{~mm}$ first $1 \mathrm{~h}$ [Westergren]) and C-reactive protein level $(78 \mathrm{mg} / \mathrm{L})$ were noted. Cardiac assessment, including electrocardiography, serial cardiac enzymes and transthoracic echocardiography, was unremarkable.

A chest $\mathrm{x}$-ray (Figure 1) revealed homogenous opacification of the left hemithorax with contralateral mediastinal shift suggesting massive pleural effusion. The left ninth rib could not be visualized and a retrosternal soft tissue opacity was noted on the lateral view. Diagnostic ultrasound-guided thoracentesis revealed a heavily blood-stained exudate $(\mathrm{pH} 7.49$, glucose $3.4 \mathrm{mmol} / \mathrm{L}$, protein $60 \mathrm{~g} / \mathrm{L}$, lactate dehydrogenase $968 \mathrm{IU} / \mathrm{L}$ and pleural fluid/blood hematocrit $>50 \%$ ). A chest tube was subsequently inserted. Cultures of pleural fluid for pyogenic bacteria, mycobacteria and fungi were negative. Cytological examination did not reveal malignant cells. A polymerase chain reaction assay for tuberculosis was negative. Bronchoscopy showed no endobronchial pathology. Serologies for rheumatological and vasculitic diseases were unremarkable.

A computed tomography scan of the chest (Figure 2) revealed two soft tissue masses with bone destruction in the region of the right fourth rib anteriorly and the left ninth rib posteriorly. These masses showed heterogeneous postcontrast enhancement. In addition, multiple welldefined lytic lesions were noted in the right scapula, sternum and body of the T4 vertebra. The findings of massive unilateral effusion and passive lung collapse were also confirmed. The mediastinal lymph nodes, vasculature and pulmonary parenchyma on the right side, along with abdominal computed tomography scan, were all unremarkable.

Fine-needle aspiration cytology (FNAC) of the soft tissue mass in the region of the left ninth rib revealed mixed cellular infiltrate consisting of abundant neutrophils, admixed with lymphocytes and several plasma cells (Figure 3). On immunocytochemistry, these cells were CD38 and CD138 positive.

The diagnosis of MM, suggested by FNAC findings of plasma cells, was subsequently confirmed with findings of multiple osteolytic lesions in the skull, lumbosacral vertebrae and pelvic bones on radiological skeletal survey; monoclonal spike in the region of gamma globulin on serum protein electrophoresis; elevated immunoglobulin (Ig) G levels

${ }^{1}$ Division of Respirology; ${ }^{2}$ Division of Hematology; ${ }^{3}$ Department of Radiology, Al-Adan Hospital, Ahmadi, Kuwait

Correspondence: Dr Mohammed H AlShati, Division of Respirology, Al-Adan Hospital, Ahmadi 64020, PO Box 46969, Kuwait.

Telephone 965-99847409, fax 965-23968915,e-mail dr_alshatti@hotmail.com 


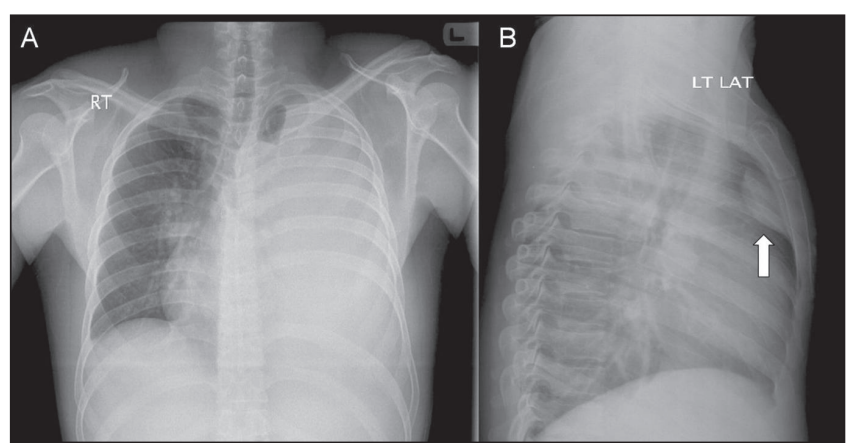

Figure 1) A Frontal chest radiograph showing almost complete opacification of the left hemithorax with contralateral mediastinal shift and total absence of the left ninth rib. B Retrosternal opacification is noted on the lateral view (arrow)

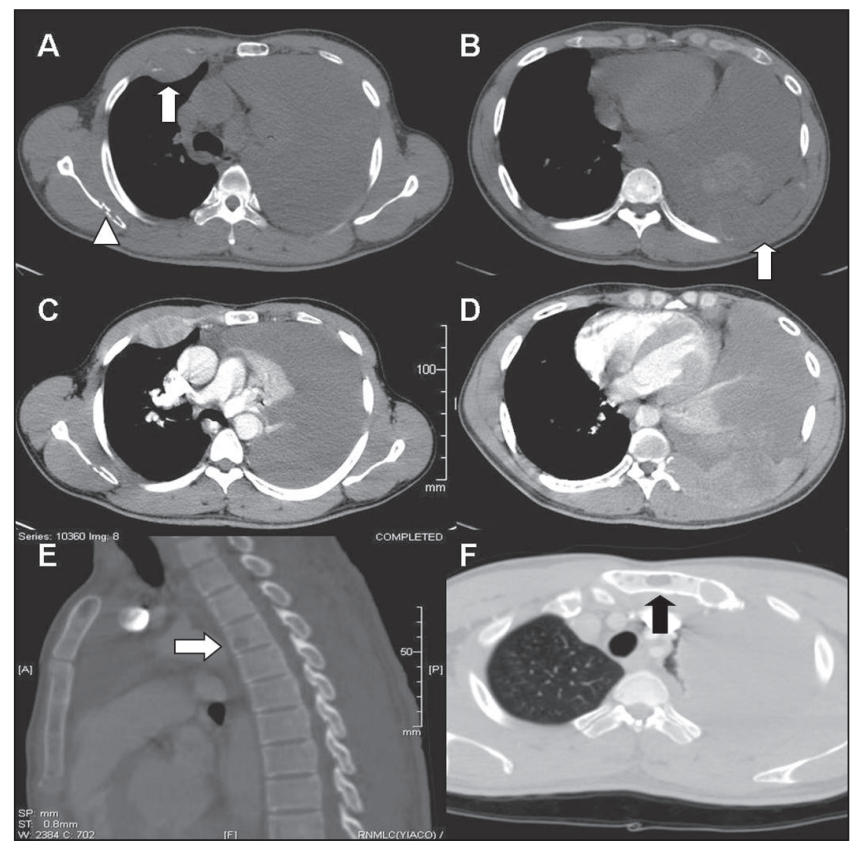

Figure 2) A Plain axial computed tomography (CT) image in the bone window demonstrates a soft tissue mass in the anterior aspect of the right fourth rib (arrow) and an area of osteolysis within the right scapula (arrow head). Massive unilateral pleural effusion is also noted. B Axial plain CT image at a lower level shows a destroyed left ninth rib posteriorly with soft tissue component (arrow) and areas of hyperdensity within the effusion in keeping with hemorrhagic nature. $\mathbf{C}$ and $\mathbf{D}$ Heterogenous postcontrast enhancement of the soft tissue masses is demonstrated. E A sagittally reformatted CT in the bone window demonstrates a lytic lesion in the body of the T4 vertebra (arrow). F Another lytic lesion is seen in the manubrium of the sternum (arrow)

$(24.5 \mathrm{~g} / \mathrm{L})$ on Ig assay that was found to be monoclonal with kappa light chains on immunofixation; reduced normal nonclonal Ig levels; Bence-Jones proteinurea consisting of kappa light chains; and increased proportion of CD38- and CD138-positive plasma cells (15\%) including immature myeloma cells on bone marrow examination.

The patient underwent melphalan chemotherapy and demonstrated good response. The chest tube was successfully removed without the need for additional pleural interventions. Follow-up at one year showed residual plasma cell tumour; the patient opted for watchful waiting and showed no signs of effusion recurrence (Figure 4).

Clinical

\section{DISCUSSION}

MM accounts for nearly $10 \%$ of all hematological malignancies and is a disorder commonly encountered in elderly patients, with a

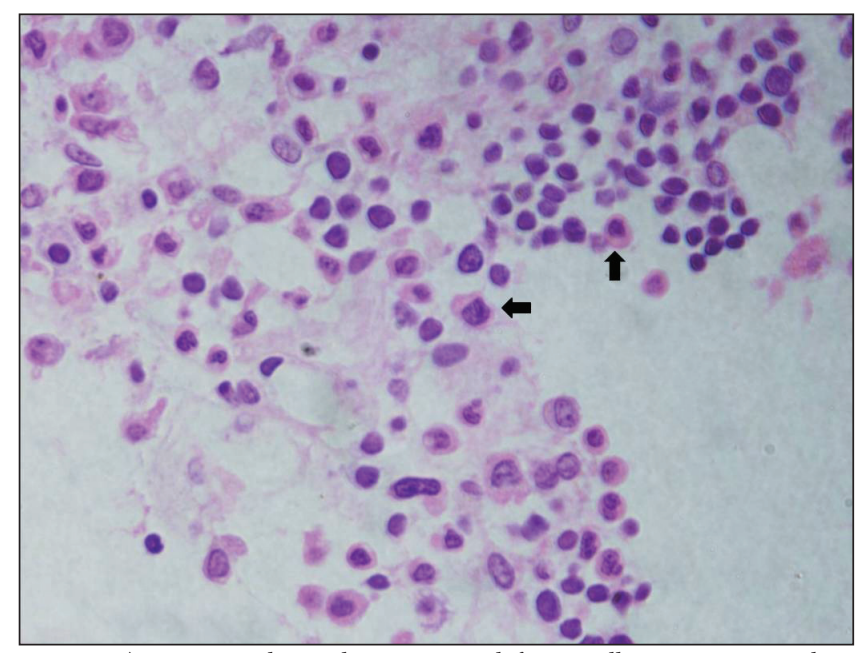

Figure 3) Hematoxylin and eosin-stained fine-needle aspiration cytology smear (original magnification $\times 400$ ) showing mixed cellular infiltrate consisting of neutrophils, lymphocytes and immature plasma cells (arrows)

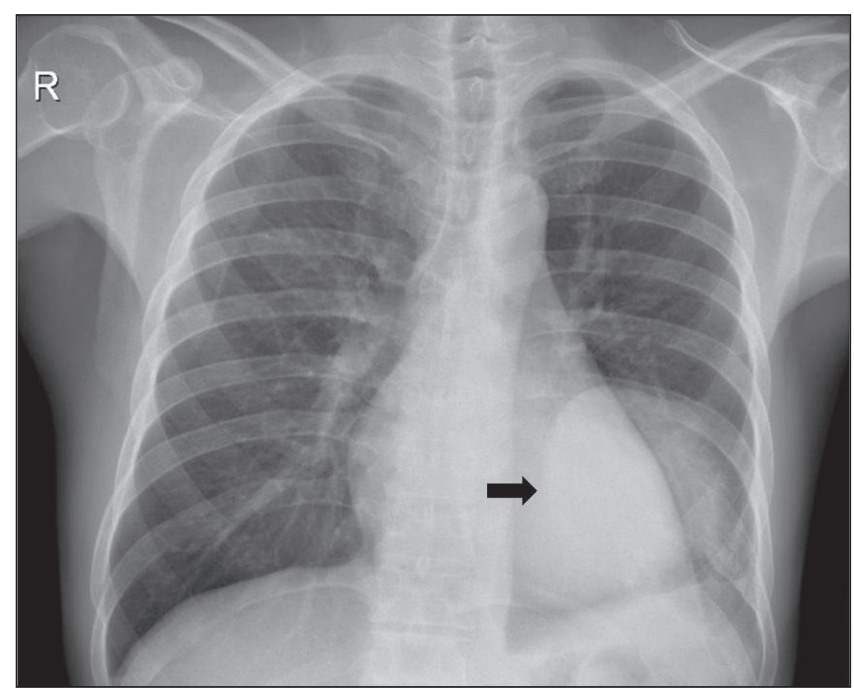

Figure 4) Follow-up chest radiograph showing minimal left pleural effusion and a well-defined soft tissue opacity (arrow) corresponding to residual plasma cell tumour of the posterior chest wall. Osteolysis of the left ninth rib is also evident

median age of 62 years. Only $2 \%$ of patients have been reported to be $<40$ years of age (2). Our patient fulfilled the established criteria for the diagnosis of MM (3) and had no familial predisposition, which can occasionally be present in younger age groups.

Pleural effusion occurs in nearly $6 \%$ of patients with MM (4). It is usually related to secondary causes such as congestive heart failure due to amyloidosis, nephrotic syndrome, pulmonary embolism, secondary neoplasm or tumour-related lymphatic obstruction. Effusion due to primary myelomatous infiltration is rare and estimated to occur in $<1 \%$ of cases (5). Pleural fluid cytology establishes a diagnosis in $>90 \%$ for myelomatous effusion (6), but has a relatively lower diagnostic yield for secondary causes. Other means of confirming myelomatous etiology include demonstration of monoclonal protein on pleural fluid electrophoresis or histological confirmation with pleural biopsy. Persistant effusion with unclear mechanism warrants pleural biopsy. Given patchy pleural involvement, pleuroscopy is preferred over closed pleural biopsy in obtaining representative samples. Pleuroscopy also has the advantage of allowing effusion drainage and pleurodesis for intractable or rapidly accumulating effusion. 
We believe that the effusion in the present case was not myelomatous but consequent to rib destruction leading to intrapleural bleeding. This was supported by normal pleural fluid protein electrophoresis, the absence of CD138-positive plasma cells in the fluid and the favourable outcome. Furthermore, none of the other secondary causes appears to be plausible. Pleural biopsy, of course, could have supported or refuted this theory but was deemed unnecessary.

\section{Radiological}

On plain imaging, it is not uncommon for physicians to notice the completely opacified hemithorax and miss the rib absence. The retrosternal opacity highlights the importance of lateral projections.

MM most commonly presents as multiple 'punched-out' osteolytic lesions involving the axial skeleton. A degree of postcontrast enhancement in the soft tissue component is not uncommon (7). Focal or diffuse rib destruction on imaging has a broad differential diagnosis and is more likely to be caused by metastatic rather than primary bone tumours. Metastatic origins associated with lytic rib lesions commonly include the lung, breast, thyroid and kidney. MM, bone sarcoma and lymphoma are among the primary malignant conditions in which bone affection is usually evident (8). Specific radiological findings, such as ground-glass patterns in fibrous dysplasia, trabecular patterns in hemangioma, calcification in osteochondroma and fluid levels in aneurysmal bone cyst, usually aid in identifying benign bone tumours (9). It should be emphasized that there is always a concern regarding the use of intravenous contrast media and kidney injury in patients with suspected myeloma.

\section{Pathological}

Except for the presence of a few suspicious immature plasma cells on FNAC, the diagnosis of MM would have been delayed or missed altogether. These cells exhibit loss of cartwheel pattern of nuclear chromatin, perinuclear halo and cytoplasmic basophilia. On immunocytochemistry, these cells stain positive with CD38 and CD138, which are markers for myeloma cells. The bone marrow examination

\section{REFERENCES}

1. Kintzer JS Jr, Rosenow EC III, Kyle RA. Thoracic and pulmonary abnormalities in multiple myeloma: A review of 958 cases. Arch Intern Med 1978;138:727-30.

2. Raab MS, Podar K, Breitkreutz 1, Richardson PG, Anderson KC. Multiple myeloma. Lancet 2009:347:324-39.

3. Kyle RA. Criteria for diagnosis of multiple myeloma. Hematol Oncol Clinic North Am 1992;6:347-58.

4. Alexandrakisk M, Passamk F, Kyriakou D, et al. Pleural effusion in hematologic malignancies. Chest 2004;125:1546-55.

5. Fekih L, Fenniche S, Hassene H, et al. Multiple myeloma presenting with multiple thoracic manifestations. Indian J Chest Dis Allied Sci 2010;52:47-9. in the present case revealed an increased proportion of plasma cells (15\%) staining positive for the above markers, further confirming the diagnosis.

\section{CONCLUSION}

The present case highlights unusual features of MM, presenting at a relatively young age with massive spontaneous hemothorax and multiple thoracic masses completely obscuring rib shadow on plain chest imaging. It is also noteworthy that hypercalcemia and renal impairment were absent. The present case serves to illustrate the need to include $\mathrm{MM}$ in the differential diagnosis of chest wall masses with underlying rib destruction, even in younger age groups.

\section{Post-test:}

1. What are causes of pleural effusion in MM?

Pleural effusion is usually related to secondary causes such as congestive heart failure due to amyloidosis, nephrotic syndrome, pulmonary embolism, secondary neoplasm or tumour-related lymphatic obstruction. Effusion due to primary myelomatous infiltration is rare and may require special confirmatory testing.

2. Which malignant conditions commonly affect the ribs? Common metastatic origins include the lung, breast, thyroid and kidney. MM, lymphoma and bone sarcoma are among the primary malignant conditions that can be associated with rib lesions.

AUTHOR CONTRIBUTIONS: All authors participated in the preparation and review of the manuscript.

DISCLOSURE: The case was presented by Dr AlShati during the 'Clinical Case Puzzlers' session at the Gulf Thoracic Society Conference (March 16 to 19, 2011, Dubai, United Arab Emirates). The authors have no financial disclosures or conflicts of interest to declare.

PATIENT PERMISSION FOR PUBLICATION: Consent was obtained from the patient for medical journal publication.

6. Meoli A, Willsie S, Fiorella R. Myelomatous pleural effusion. South Med J 1997;90:65-8.

7. O'Sullivan P, O'Dwyer H, Flint J, Munk PL, Muller NL. Malignant chest wall neoplasms of bone and cartilage: A pictorial review of CT and MR findings. Br J Radiol 2007;80:678-84

8. Guttentag AR, Salwen JK. Keep your eyes on the ribs: The spectrum of normal variants and disease that involve the ribs. RadioGraphics 1999;19:1125-42.

9. De maeseneer M, De mey J, Lenchik 1, Everaert H, Osteaux M. Helical CT of rib lesions: A pattern-based approach. AJR Am J Roentgenol 2004;182:173-9. 


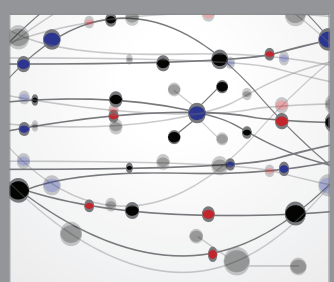

The Scientific World Journal
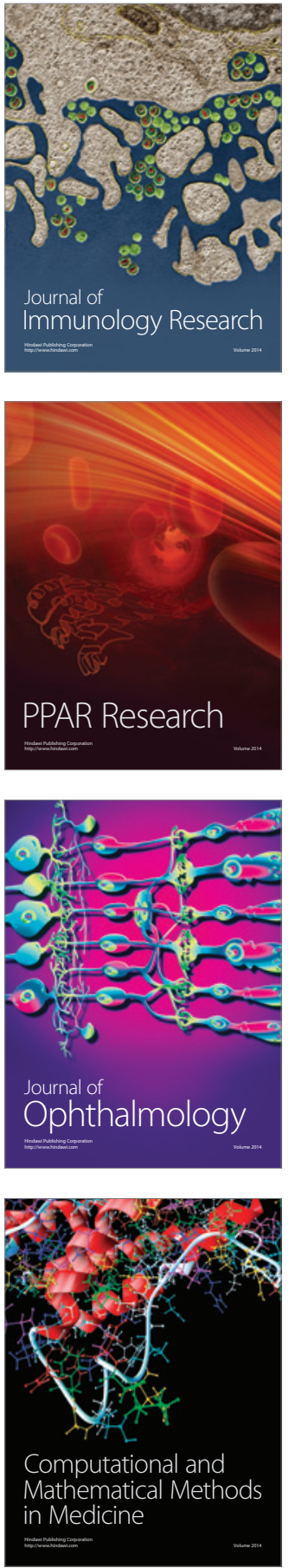

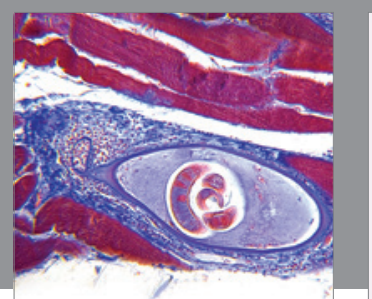

Gastroenterology Research and Practice

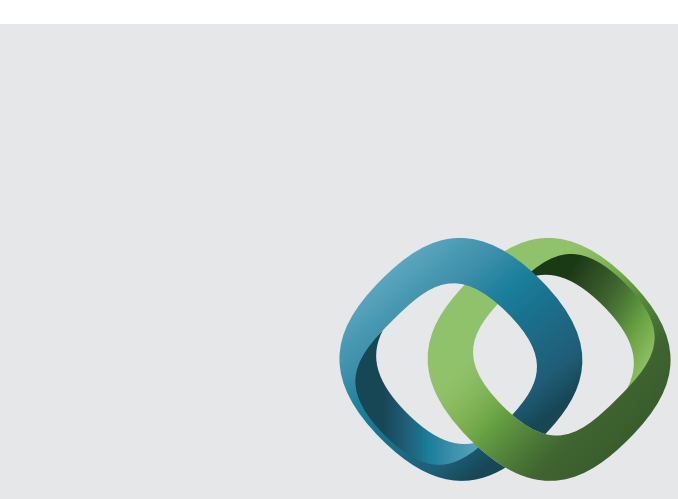

\section{Hindawi}

Submit your manuscripts at

http://www.hindawi.com
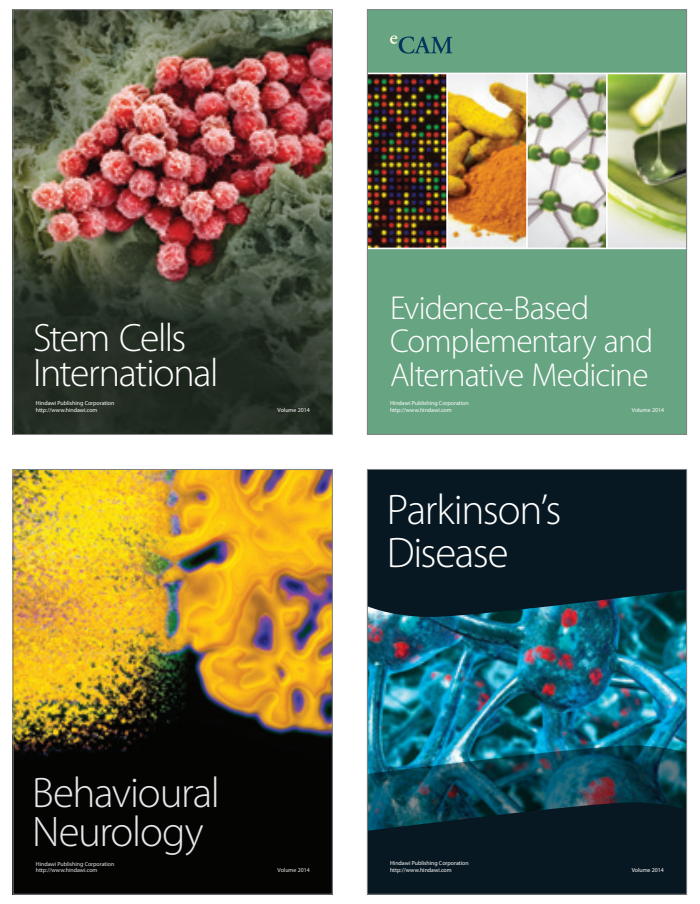
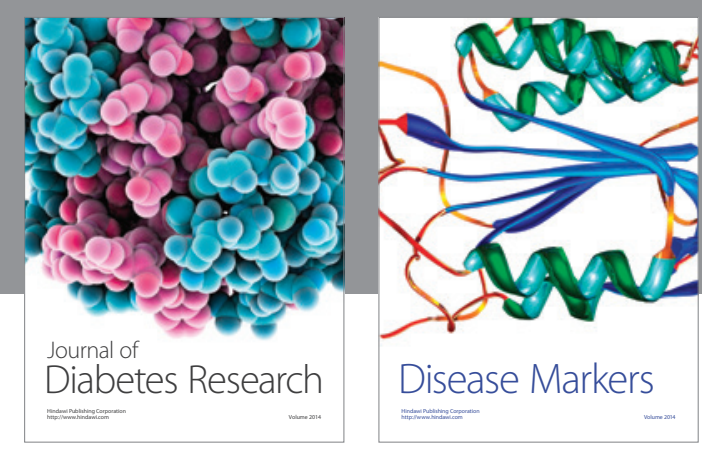

Disease Markers
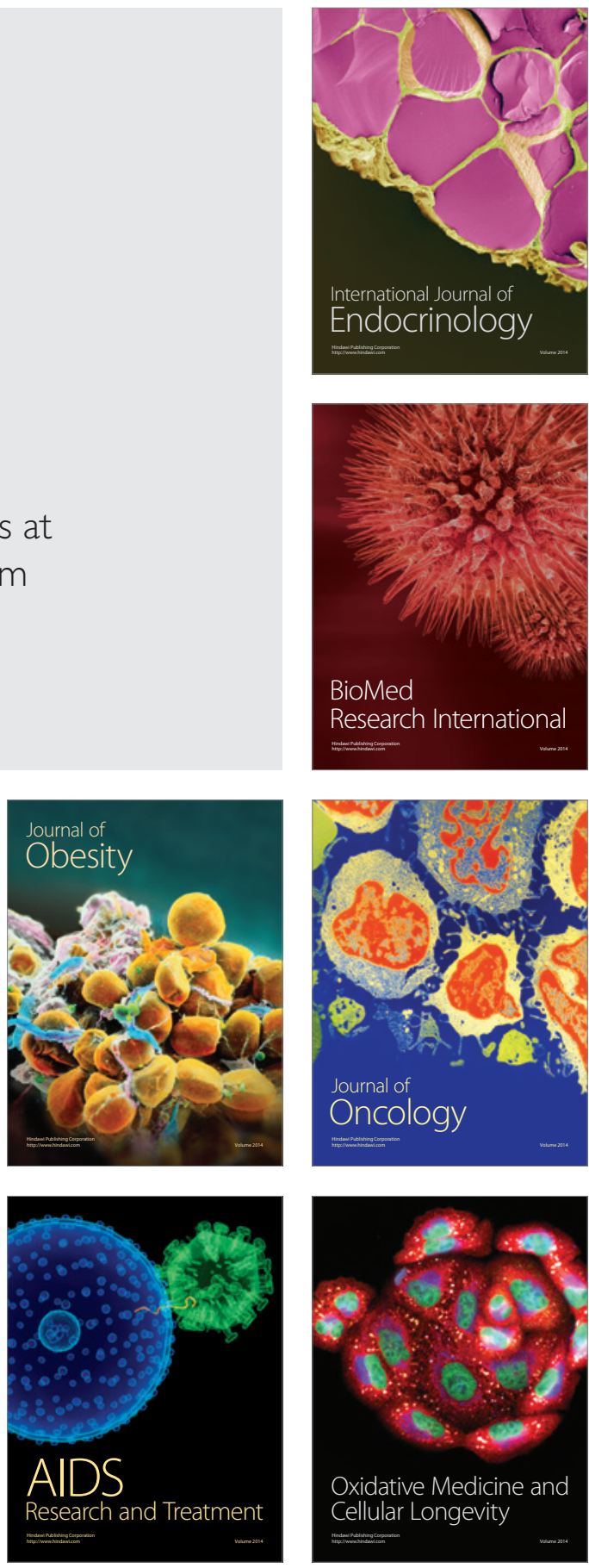